\title{
Isolated tricuspid valve surgery in patients with previous cardiac surgery
}

\author{
Bettina Pfannmüller, MD, Monica Moz, MD, Martin Misfeld, MD, PhD, Michael A. Borger, MD, PhD, \\ Anne-Kathrin Funkat, PhD, Jens Garbade, MD, PhD, and Friedrich W. Mohr, MD, PhD
}

\begin{abstract}
Objectives: Few studies have been published in literature on outcomes of isolated tricuspid valve (TV) surgery when performed as a reoperation. Hence, we analyzed our early and midterm results of TV surgery in this unique group of patients.
\end{abstract}

\begin{abstract}
Methods: We performed a retrospective analysis of 82 consecutive patients who underwent isolated TV surgery as a reoperation at our institution between 1997 and 2010. Symptomatic TV regurgitation (84.2\%), acute endocarditis $(14.6 \%)$, and valve thrombosis after TV repair $(1.2 \%)$ were the indications for surgery. A minimally invasive access through a right anterolateral thoracotomy was the preferred approach in $60 \%$ of the patients. Previous cardiac operations included mitral, aortic, and TV surgery in $60 \%, 29 \%$, and $27 \%$ and coronary bypass surgery in $18 \%$, usually performed as combined procedures. Elective surgery was performed in $67.1 \%$ of the patients. Mean patient age was $64.1 \pm 11.9$ years, $28 \%$ being male with an average logistic EuroSCORE of $16.4 \% \pm 14.3 \%$. Follow-up was $96 \%$ complete, with a mean duration of $2.6 \pm 2.4$ years.
\end{abstract}

Results: Overall thirty-day mortality was $14.6 \%$; for patients without and with endocarditis, it was $12.9 \%$ and $25 \%$. Thirty-day mortality for patients undergoing elective surgery was $4.0 \%$. Overall 2 -year survival was $63.0 \% \pm 5.5 \%$. The 2-year freedom from TV-related reoperation was $93.5 \% \pm 3.3 \%$.

Conclusions: Postoperative results of isolated TV surgery as a reoperation are acceptable when performed electively but dismal in patients undergoing nonelective surgery. Thus, redo TV surgery, when indicated, should be performed sooner rather than later. Minimally invasive surgery through a right lateral minithoracotomy is a safe approach for patients with elective surgery. (J Thorac Cardiovasc Surg 2013;146:841-7)

Isolated tricuspid valve (TV) surgery, especially as a reoperation, is considered to be associated with high operative risk. Although the operation may not be technically complicated, the increased risk is usually due to the fact that patients are referred for surgery late in their disease process. Such patients often have evidence of right heart failure and associated complications. It is unknown whether poor postoperative outcome is related to the severity of tricuspid regurgitation (TR) itself or to the poor overall status of such patients. In addition, patients with isolated TV disease and previous cardiac surgery represent an even more challenging subgroup. Precise clinical outcomes for these challenging patients still need to be determined.

Only a handful of publications regarding outcome of patients with TV surgery as a reoperation have been published so far, usually with small patient numbers. ${ }^{1-4}$ The results of these studies are not uniform because of different patient

\footnotetext{
From the Department of Cardiac Surgery, Heart Center, University of Leipzig, Leipzig, Germany.

Disclosures: Authors have nothing to disclose with regard to commercial support.

Received for publication Jan 29, 2012; revisions received June 30, 2012; accepted for publication July 31, 2012; available ahead of print Aug 30, 2012.

Address for reprints: Bettina Pfannmüller, MD, Department of Cardiac Surgery, University of Leipzig-Heart Center, Strümpellstrasse 39, 04289 Leipzig, Germany (E-mail: pfab@med.uni-leipzig.de).

$0022-5223 / \$ 36.00$

Copyright (C) 2013 by The American Association for Thoracic Surgery http://dx.doi.org/10.1016/j.jtcvs.2012.07.096
}

cohorts and differences in valve disease. In these studies, hospital mortality ranged from $0 \%$ to $37 \% .^{1-4}$

The optimal timing for surgery and the best surgical strategy in these challenging patients remains unclear. The purpose of this study was to review our single-center experience of patients undergoing isolated TV surgery after previous cardiac surgery in a relatively large number of patients.

\section{PATIENTS AND METHODS}

Between March 1997 and December 2010, a total of 82 patients underwent isolated TV repair or replacement as a reoperation at the Heart Center Leipzig.

Indications for TV surgery were symptomatic TR in 69 (84.2\%) patients; of these, the main TV disease was annulus dilatation in 49 $(61.0 \%)$ patients, restriction of the leaflets in $12(14.7 \%)$ patients, rolled up and thickened leaflets in $3(3.7 \%)$ patients, a dehiscence of the previously implanted ring in $2(2.4 \%)$ patients, an atrophic TV in $1(1.2 \%)$ patient, and resection of the TV in the previous surgery owing to massive endocarditis in $1(1.2 \%)$ patient. In combination with the previously described pathologic conditions were fenestrations of and adherences with the leaflets owing to a pacemaker lead additionally responsible for TR in $8(9.8 \%)$ patients.

Active endocarditis was the reason for TV surgery in $12(14.6 \%)$ patients: intravenous drug abuse in 3 of these and vegetations on permanent pacemaker leads with involvement of the TV in another 3 patients. In the remaining 6 patients, the cause of endocarditis was unknown.

A pannus combined with thrombosis based on the previous implanted tricuspid ring after TV repair resulting in TV stenosis was the reason for repeat TV surgery in $1(1.2 \%)$ patient. 


$$
\begin{aligned}
& \text { Abbreviations and Acronyms } \\
& \begin{aligned}
\text { CI } & =\text { confidence interval } \\
\text { CPB } & =\text { cardiopulmonary bypass } \\
\text { NYHA } & =\text { New York Heart Association } \\
\text { TR } & =\text { tricuspid regurgitation } \\
\text { TV } & =\text { tricuspid valve }
\end{aligned}
\end{aligned}
$$

Patients without ( $\mathrm{n}=70$, group 1$)$ and with $(\mathrm{n}=12$, group 2$)$ pannus were divided into 2 groups on the basis of the different pathologic conditions, which could complicate the analysis.

In $55(67.1 \%)$ patients, the operation was performed electively. In the remaining patients, the operation was performed "nonelectively." "Nonelective" surgery was performed in the 12 patients with active endocarditis, in 10 patients with preoperative global cardiac decompensation, in 4 patients with right heart decompensation, and in the patient with a pannus combined with thrombosis based on the previously implanted tricuspid ring after TV repair.

Preoperative, intraoperative, and postoperative parameters of all patients were prospectively entered into a patient data management system and then retrospectively analyzed. In addition, further chart review and information from preoperative and predischarge echocardiographic reports were collected and analyzed. TR was calculated by measurement of vena contracta in a 4-chamber view. ${ }^{5}$

TV surgery was performed using standard techniques including bicaval cannulation and mild hypothermic cardiopulmonary bypass (CPB). Patients were operated on via sternotomy $(\mathrm{n}=36,43.9 \%)$ or minimally invasive access $(n=46,56.1 \%)$. The minimally invasive technique via a right anterolateral minithoracotomy has been described by our group before. $^{6}$

Surgery was performed either with aortic crossclamping and cardioplegic arrest $(\mathrm{n}=20 ; 24 \%)$ or without crossclamping and cardioplegia either on the beating heart $(\mathrm{n}=57 ; 70 \%)$ or with a short period of ventricular fibrillation $(\mathrm{n}=5 ; 6 \%)$.

TV repair or replacement was performed depending on the pathologic condition of the valve and the surgeon's choice. The surgical access, as well as the decision to perform the operation with or without aortic crossclamping, was the surgeon's choice as well.

Ethics approval was granted from the local ethics committee for this investigation.

\section{Follow-up}

After discharge, all patients were contacted by mail and asked to answer a specific questionnaire on an annual basis. Patients who did not respond were contacted by telephone. If no further information about the patients was available, family physicians were contacted directly. Follow-up was $97 \%$ complete with a mean follow-up time of $2.6 \pm 2.4$ years (range, 1 day-11.3 years).

With patient agreement, we additionally contacted the patient's cardiologist to obtain the most recently performed postoperative echocardiographic report, which was available in $45 \%$ of the surviving patients at a mean follow-up time of $3.2 \pm 2.2$ years (range, 35 days-9.4 years).

\section{Statistical Evaluation}

Results are displayed in the standard format with continuous variables expressed as mean \pm standard deviation and categorical data as proportions throughout the manuscript. Risk analysis was done with binary logistic regression analysis. Cumulative survival, as well as the freedom from valve-related reoperation, was calculated by Kaplan-Meier methods. Differences in follow-up were calculated with $95 \%$ confidence limits and compared by log-rank (Mantel-Cox) test.
All statistical analyses were performed using SPSS statistical package 17.0 (SPSS Inc, Birmingham, Ala).

\section{RESULTS}

Overall, 82 patients underwent isolated TV surgery as a reoperation during the study period. Mean age of all patients was $64.1 \pm 11.9$ years (range, $22-83$ years). In the whole cohort were 23 male patients $(28 \%)$. Average New York Heart Association (NYHA) class was $2.6 \pm 0.7$. Left ventricular function was normal in the majority of patients and atrial fibrillation was present in $29(35.4 \%)$ patients. Table 1 shows the demographic data of all patients undergoing isolated TVoperation, as well as those of groups 1 and 2 (ie, patients without and with endocarditis).

There were significantly more patients with atrial fibrillation and arterial hypertension in group 1 than in group 2.

In a total of $55(67.1 \%)$ patients, surgery was performed electively.

In $20(24.4 \%)$ patients, a previous TV operation was performed; 16 of these operations were TV repair. Figure 1 displays the previous cardiac procedures, broken down into the 2 patient groups. Previous left-sided surgery was performed in $55(67.1 \%)$ patients.

Preoperative echocardiography showed severe TR $3+$ to $4+$ in $76(92 \%)$ patients, in 68 patients of group 1 and in 8 patients of group 2 . TR $2+$ was present in $3(4 \%)$ patients, in 1 of group 1 and 2 of group 2. Mild or even no TR at the time of surgery was present in $3(4 \%)$ patients: 1 patient of group 1 had a thrombus with secondary tricuspid stenosis after previous TV repair, 1 patient (group 2) had endocarditis of a mechanical tricuspid prostheses, and 1 patient (group 2) had a vegetation of an implantable cardioverter defibrillator lead with involvement of the TV without significant TR. There were significant differences in the distribution of preoperative TR in both groups $(P<.01)$.

Intraoperative and perioperative data are shown in Table 2. Operation, crossclamp, and CPB times were $174 \pm 66$ minutes, $57 \pm 28$ minutes, and $101 \pm 49$ minutes and were comparable in patients with and without endocarditis. Intraoperative procedures are shown in Figure 2.

Minimally invasive surgery through a right lateral minithoracotomy with femoral cannulation for CPB was used in $42(60.0 \%)$ of the patients in group 1 and in $4(33.3 \%)$ of the patients in group 2. TV surgery in the remaining patients was performed via median sternotomy.

There were significantly shorter operation and crossclamp times for patients undergoing minimally invasive surgery compared with patients with sternotomy (operation time, $168 \pm 56$ minutes vs $183 \pm 79$ minutes; $P=.03$; crossclamp time, $27 \pm 18$ minutes vs $48 \pm 38$ minutes; $P=.02)$ and comparable CPB times (103 \pm 47 minutes vs $94 \pm 54$ minutes; $P=.5)$. Operation, crossclamp, and CPB times were 171 \pm 66 minutes, $57 \pm 28$ minutes, and $101 \pm 49$ minutes and were comparable in patients with and without endocarditis. 
TABLE 1. Demographic patient data

\begin{tabular}{|c|c|c|c|c|}
\hline & All patients $(n=82)$ & No endocarditis $(n=70)$ & Endocarditis $(n=12)$ & $P$ value \\
\hline Age (y) & $64.12 \pm 11.9$ & $65.2 \pm 10.9$ & $57.9 \pm 16.0$ & .15 \\
\hline Male & $23(28.0 \%)$ & $18(25.7 \%)$ & $5(41.7 \%)$ & .3 \\
\hline Atrial fibrillation & $29(35.4 \%)$ & $27(38.6 \%)$ & $2(16.7 \%)$ & .01 \\
\hline Arterial hypertension & $50(61.0 \%)$ & $45(64.3 \%)$ & $5(41.7 \%)$ & .03 \\
\hline Diabetes mellitus & $16(19.5 \%)$ & $14(14.8 \%)$ & $2(16.7 \%)$ & 6 \\
\hline Body mass index & $25.2 \pm 4.2$ & $24.8 \pm 3.6$ & $28.0 \pm 6.1$ & .1 \\
\hline Preop NYHA & $2.6 \pm 0.7$ & $2.6 \pm 0.6$ & $2.5 \pm 1.2$ & .9 \\
\hline logEuroSCORE & $16.4 \% \pm 14.3 \%$ & $15.1 \% \pm 12.7 \%$ & $24.2 \% \pm 20.7 \%$ & .17 \\
\hline LVEF & $50.7 \% \pm 23.5 \%$ & $56.7 \% \pm 13.7 \%$ & $61.2 \% \pm 10.4 \%$ & .3 \\
\hline Pulmonary artery pressure & $43.8 \pm 21.8$ & $43.5 \pm 21.5$ & $46.5 \pm 29.1$ & .7 \\
\hline No. of thrombocytes & $202.0 \pm 90.9$ & $205.9 \pm 94.1$ & $180.6 \pm 70.0$ & .4 \\
\hline Permanent Pacemaker/AICD & $34(41.4 \%)$ & $31(44.3 \%)$ & $3(25.0 \%)$ & .4 \\
\hline TV reoperation & $20(24.4 \%)$ & $16(122.9 \%)$ & $4(33.3 \%)$ & .09 \\
\hline Previous left-sided surgery & $58(72.5 \%)$ & $52(76.5 \%)$ & $6(50.0 \%)$ & .06 \\
\hline
\end{tabular}

Preop NYHA, Preoperative New York Heart Association; logEuroSCORE, logistic EuroSCORE predicted risk of operative mortality; LVEF, left ventricular ejection fraction; $A I C D$, automatic implantable cardioverter defibrillator; $T V$, tricuspid valve.

The tricuspid procedure was performed with a crossclamp and cardioplegic arrest in $20(24 \%)$ patients. In the remaining 62 patients, surgery was done with the heart beating $(\mathrm{n}=57,70 \%)$ or during ventricular fibrillation $(\mathrm{n}=5,6 \%)$.

A mechanical $(n=7)$ or biological $(n=13)$ TV replacement was performed in $20(24 \%)$ patients. These operations were performed owing to excessive retraction of the leaflets in 11 patients, thickened and rolled up leaflets in 3 patients, active endocarditis in 3 patients, a previously resected TV because of endocarditis in 1 patient, atrophic leaflets in 1

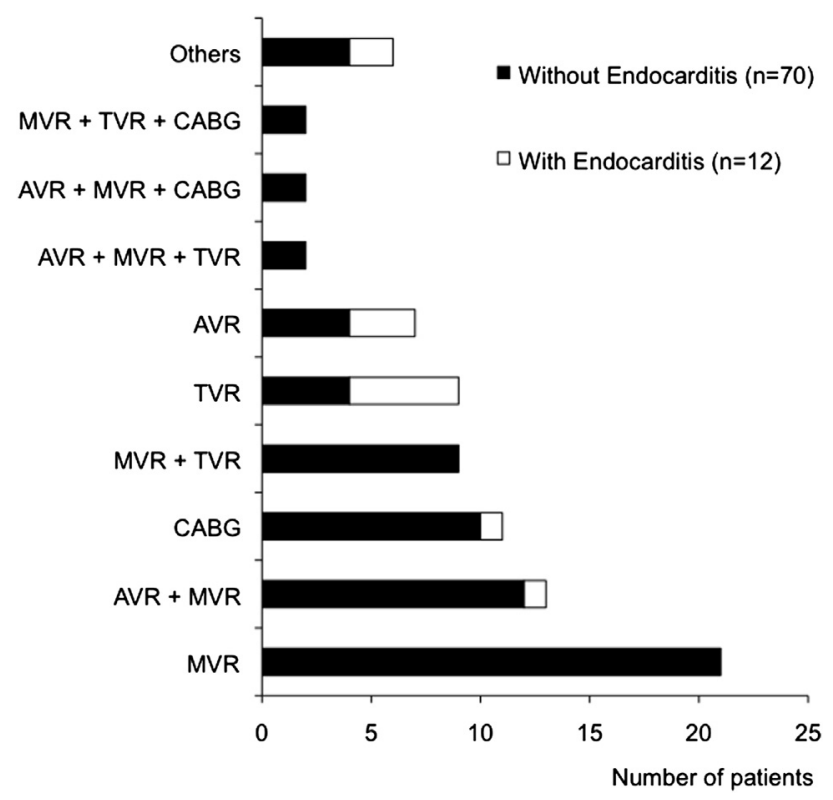

FIGURE 1. Surgical procedures performed in patients with and without endocarditis. $M V R$, Mitral valve repair/replacement; $T V R$, tricuspid valve repair/replacement; $C A B G$, coronary artery bypass grafting; $A V R$, aortic valve repair/replacement. patient, and excessive pannus and thrombus after TV repair in 1 patient.

In this patient group were included 8 of the 16 patients with previous TV repair.

TV repair was performed in the remaining $62(76 \%)$ patients. Of these, 8 of 16 patients had a previous TV repair.

Residual TR $3+$ was present in $5(8.0 \%)$ patients and predischarge TR $2+$ in $9(12.3 \%)$ patients, in all cases after TV repair. No patient with preoperative endocarditis had residual moderate or more TR.

TR $2+/ 3+$ was caused by central regurgitation in all 14 patients. A preoperatively inserted permanent pacemaker lead was suspected to be the cause of the central TR in 3 of the 14 patients.

Reoperation owing to bleeding complications was necessary in a total of $12(14.6 \%)$ patients, $7(8.5 \%)$ with postoperative active bleeding and $5(6.1 \%)$ with relevant pericardial or pleural hematoma that developed some days after the operation.

Postoperative low cardiac output syndrome occurred in $12(14.6 \%)$ patients. Four $(4.9 \%)$ were treated with extracorporal membrane oxygenation and $3(3.7 \%)$ with an intraaortic balloon pump.

Postoperative implantation of a permanent pacemaker was necessary in $7(8.5 \%)$ patients. We did not observe a significant difference in pacemaker requirements for patients who underwent TV repair versus TV replacement $(P=.2)$.

New postoperative permanent neurologic deficits were detected in $4(4.9 \%)$ patients, during postoperative extracorporal membrane oxygenation in 2 of them (1 patient with cerebral edema and 1 patient with fulminant cerebral bleeding). The 2 remaining patients had previously implanted mechanical mitral and mechanical mitral and aortic valve replacement, respectively, and on computed 
TABLE 2. Perioperative patient characteristics

\begin{tabular}{|c|c|c|c|c|}
\hline & All patients $(n=82)$ & No endocarditis $(n=70)$ & Endocarditis (n = 12) & $P$ value \\
\hline Operation time (min) & $174.3 \pm 66.3$ & $171.0 \pm 60.1$ & $188.8 \pm 97.3$ & .4 \\
\hline Patients operated on with crossclamp & $20(24.4 \%)$ & $15(21.4 \%)$ & $5(41.7 \%)$ & .3 \\
\hline Crossclamp time (min) & $57.0 \pm 28.3$ & $52.3 \pm 25.1$ & $70.0 \pm 36.4$ & .3 \\
\hline CPB time (min) & $100.9 \pm 48.7$ & $101.9 \pm 45.8$ & $95.3 \pm 64.8$ & .4 \\
\hline Minimally invasive access & $46(56.1 \%)$ & $42(60.0 \%)$ & $4(33.3 \%)$ & .09 \\
\hline Preop TR & $3.2 \pm 0.7$ & $3.4 \pm 0.6$ & $2.5 \pm 1.1$ & .02 \\
\hline Postop TR & $0.9 \pm 0.9$ & $0.9 \pm 0.9$ & $0.5 \pm 0.5$ & .03 \\
\hline Postop LVEF & $53.8 \pm 13.1$ & $54.0 \pm 12.7$ & $52.4 \pm 16.6$ & .7 \\
\hline
\end{tabular}

Data are mean \pm standard deviation. $C P B$, Cardiopulmonary bypass; preop/postop TR, preoperative/postoperative tricuspid regurgitation grade; postop LVEF, postoperative left ventricular ejection fraction.

tomography they had postoperative new ischemic lesions verified, resulting in a hemiparesis of the left arm and epileptic seizures, respectively.

Overall 30-day mortality was $14.6 \%$ with $9(12.9 \%)$ deaths occurring in group 1 and $3(25.0 \%)$ deaths in group $2(P=.3)$. Overall early mortality after elective surgery was $4.0 \%$ with 2 deaths; after minimally invasive surgery, early mortality was $0 \%$. Early mortality after nonelective surgery was $31.2 \%$ with 10 deaths $(P=.01)$.

We performed a binary logistic regression analysis to determine the independent risk factors for early mortality with the covariates gender, age, preoperative left ventricular ejection fraction, preoperative NYHA, body mass index, logistic EuroSCORE, priority for surgery, pulmonary hypertension, method for TV surgery (TV surgery performed with crossclamping, beating heart, or ventricular fibrillation), endocarditis, previous left-sided surgery, and approach for surgery. Only elective surgery (odds

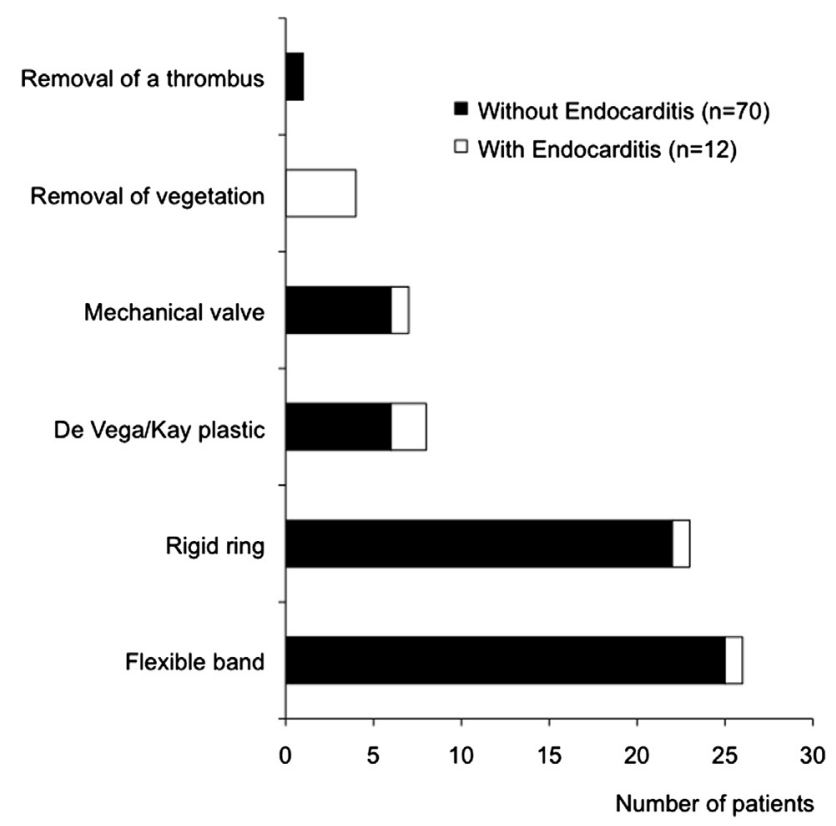

FIGURE 2. Intraoperative surgical procedures in patients with and without endocarditis. ratio, 9.8) was revealed as a protective factor for early mortality.

Midterm survival of all patients at 2 years was $63.0 \% \pm$ $5.5 \%$ (95\% confidence interval [CI], 44-73). Two-year survival was $66.0 \% \pm 5.8 \%(95 \% \mathrm{CI}, 46-99)$ in group 1 patients versus $45.0 .7 \% \pm 15.6 \%(95 \% \mathrm{CI}, 16-49)$ in group 2 (log-rank $P=.25$ ). There was a significant difference $(P<.001)$ in patients undergoing elective and nonelective surgery with 2 -year survivals of $78.5 \% \pm 6.1 \%$ (95\% CI, 55-94) and 38.4\% $\pm 8.8 \%$ (95\% CI, 28-42), as shown in Figure 3. There was no significant difference between the long-term survival of patients with persistent TR $2 / 3+$ after TR repair in regard to patients with TR $0 / 1+(P=.3)$.

Midterm freedom from TV-related reoperation is depicted in Figure 4. After 2 years, freedom from TV-related reoperation was $93.5 \% \pm 3.3 \%(95 \% \mathrm{CI}$, 98-129) for all patients, $96.8 \% \pm 2.2 \%(95 \%$ CI, 25-55) for patients without, and $67.5 \% \pm 20.7 \%(95 \% \mathrm{CI}$, 106-134) for patients with endocarditis, respectively $(P=.03)$. TV reoperation had to be performed in 7 patients between 21 days and 3 years postoperatively because of the following reasons: severe, symptomatic central TR in 2 patients, ring dehiscence with severe TR in another patient, thrombosis of a previous TV repair in 1 patient, recurrent endocarditis after TV replacement in the presence of drug abuse in 2 patients, and endocarditis in 1 patient after TV replacement with acute myeloid leukemia in his history.

Postoperative echocardiographic data with a mean follow-up time of $3.2 \pm 2.2$ years were available in 25 of the surviving patients. Worsening of the predischarge level of TR was observed 2 patients who underwent TV repair and reoperation and in 2 of 6 patients who underwent biological TV replacement. The latter patients were 36 and 38 years old at the time of surgery and had no TR on the predischarge echocardiogram, but 4 years later had TR $2+$ to $3+$.

Postoperative NYHA classification in follow-up was on average $2.4 \pm 0.9$. During follow-up, 2 bleeding complications $(0.9 \%$ /patient-year $)$ were observed with 


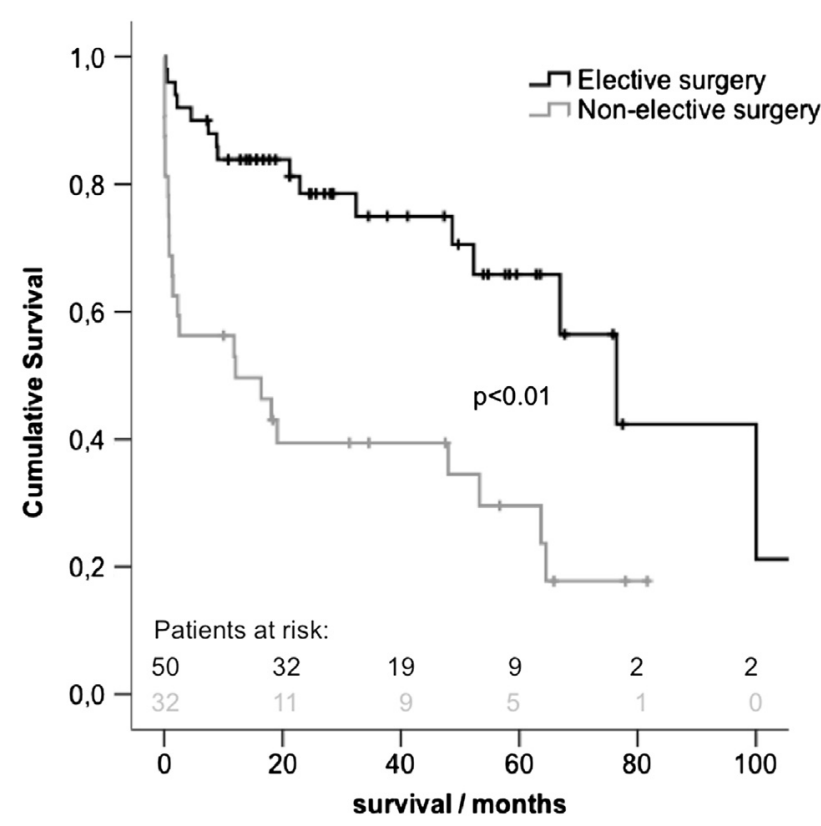

FIGURE 3. Postoperative survival for patients with elective and nonelective surgery.

hemodynamically relevant nasal bleeding under anticoagulation, both in patients with TV repair and mechanical mitral and mechanical mitral and aortic prostheses, respectively. During follow-up, new permanent pacemakers were implanted in 2 patients and 1 intravenous pacemaker lead was changed into a coronary sinus lead (1.4\%/patient-year).

We failed to find any difference between patients who received tricuspid repair or replacement with regard to 30-day

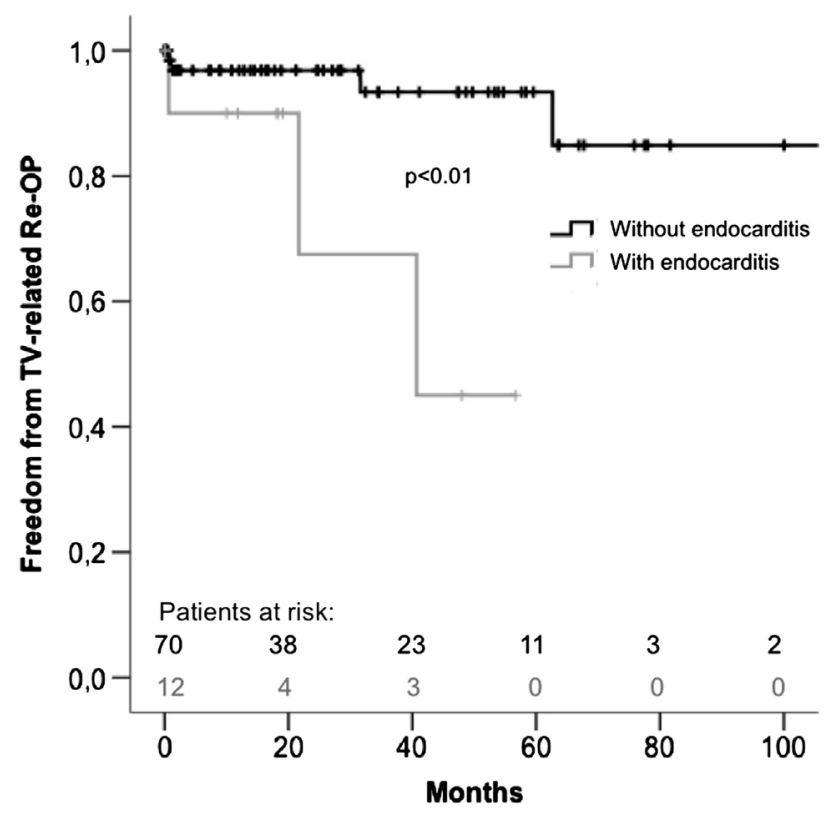

FIGURE 4. Postoperative freedom from tricuspid valve-related reoperation for patients with and without endocarditis. $T V$, Tricuspid valve. mortality, midterm survival, and freedom from TV-related reoperation.

\section{DISCUSSION}

We have shown that patients undergoing isolated TV surgery as a reoperation have a good early clinical outcome if surgery is performed as an elective procedure. Clinical outcomes are markedly worse if surgery is performed on an urgent basis owing to endocarditis or cardiac decompensation.

Our results are in agreement with those of the literature. TV reoperations owing to rheumatic TR in combination with other cardiac procedures show excellent early postoperative results with very low hospital mortality ${ }^{7,8}$ if performed as an elective procedure. On the other hand, Bernal and associates ${ }^{3}$ showed a hospital mortality of $35.1 \%$ in patients who underwent TV reoperations after previous TV repair. This high mortality rate may be related to the fact that the authors did not differentiate between patients requiring elective or emergency surgery. Although the study is not completely comparable with our data because Bernal and colleagues focused on patients with reoperation of the TV itself and we focused on patients with previous cardiac surgery and indication for isolated TV surgery, Bernal's data may give some insights into this high-risk group of patients undergoing reoperative TV surgery.

In our entire patient group, we could demonstrate a 30-day mortality rate of $14.6 \%$, reflecting the increased risk associated with reoperative TV surgery. However, we observed a large difference in mortality between patients having elective and nonelective procedures $(4.0 \%$ vs $35.7 \%$, respectively). As Figure 3 demonstrates, survival after reoperative $\mathrm{TV}$ repair is mainly related to early postoperative mortality, inasmuch as the curve of the nonelective group drops dramatically in the early postoperative course. The risk of early mortality for patients with elective surgery as compared with patients with nonelective surgery was significantly higher with an odds ratio of 10 . Two-year survival for patients with nonelective surgery was $38.4 \% \pm 8.8 \%$ (95\% CI, 28-42). In comparison, the 2-year survival of patients with conservatively treated severe TR is approximately $40 \%$, as described by Nath, Foster, and Heidenreich. ${ }^{9}$

Another interesting point is the early mortality of $0 \%$ for patients with elective minimally invasive surgery in our cohort. This observation is probably related to selection bias, inasmuch as $70 \%$ of the patients with elective and only $39 \%$ of the patients with nonelective surgery were treated in a minimally invasive fashion. That said, minimally invasive surgery could be advantageous in certain situations. To evaluate the position of the heart and exclude excessive adhesions between the right chest and lung, we routinely perform a computed tomographic scan preoperatively. If minimally invasive surgery is possible owing to expected 
few adhesions, we usually cannulate the right femoral artery and the right femoral and internal jugular veins. We introduce a Fogarty catheter through the femoral and jugular veins to block the inferior and superior venae cavae for reoperative TV procedures, which avoids the need for dissection and clamping or snaring of the venae cavae. To avoid dissection of the aorta, we usually perform the procedure on a beating heart or in ventricular fibrillation when a minimally invasive approach is used. Fukuda and associates ${ }^{10}$ use a similar cannulation technique for patients with TV reoperation performed through a median sternotomy, blocking the inferior vena cava with a Foley catheter. These authors speculate that this technique is an advantage in these patients, associated with excellent drainage with low venous pressure. In addition, this technique avoids cannulation of a dilated azygos or hepatic vein and minimizes dissection of the right atrium and ventricle, which again minimizes postoperative bleeding. We are convinced that prevention of dissection of the right ventricle, which is possible in minimally invasive surgery, is additionally protective against dilatation of the right ventricle after surgery that would result in poor right heart function.

TV repair was performed in nearly $75 \%$ of our patients. However, $17 \%$ of all repaired valves continued to have residual TR $2+$ to $3+$ at predischarge echocardiography. There was no worsening of TR in echocardiographic follow-up in those patients who had available data.

However, the intraoperative decision for TV repair or replacement in these patients is challenging. Preoperative poor right ventricular function may result in further ventricular deterioration after TV replacement when the ventricle is challenged with the whole blood volume. However, we were unable to display a difference in outcomes between patients who underwent TV repair or replacement in our cohort, although only 20 patients underwent TV replacement surgery.

Kunadian and associates ${ }^{11}$ reviewed studies regarding long-term results after mechanical and biological TV replacement and found no major differences regarding survival or reoperation rates. Our policy is to use bioprosthetic valves for TV replacement in nearly all patients, regardless of patient age or presence of a previously implanted mechanical prosthesis in the aortic and/or mitral position. We prefer biological TV replacement to avoid excessive anticoagulation, which is necessary in patients with a mechanical TV replacement owing to the low pressure in the right atrium and ventricle. On the other hand, we were surprised to find 2 young patients ( 36 and 38 years old at time of surgery) with moderate and severe TR 4 years after biological TV replacement, despite normal predischarge echocardiographic findings.

We found 3 patients with a permanent pacemaker and predischarge central TR $2+$ to $3+$ after TV repair, in whom the pacemaker lead was described to interfere with the movement of the TV on echocardiography. In none of these patients was interference of the permanent pacemaker lead with the coaptation of the TV observed intraoperatively. McCarthy and coworkers ${ }^{4}$ described transtricuspid permanent pacemaker leads as a risk factor for postoperative failure of TV repair and concluded that all permanent pacemaker leads should be removed intraoperatively and replaced epicardially. In our own experience, ${ }^{12}$ we prefer to replace permanent pacemaker leads during TV repair only if they seem to interfere with TV leaflet mobility when tested on the beating heart intraoperatively.

We routinely place an epimyocardial pacemaker lead at the time of surgery in patients undergoing TV replacement, even in those undergoing minimally invasive surgery. In TV reoperations, minimally invasive epimyocardial implantation is very difficult and results in poor sensing and pacing because of adhesions and fibrosis. We therefore place a coronary sinus lead in such patients and tunnel this lead through the atrial wall.

If a new permanent pacemaker is required in a patient with a previous TV replacement, we prefer to implant the pacemaker lead into the coronary sinus. ${ }^{13}$ Another option in patients with a bioprosthesis is the transvenous placement of a new lead directly through the prosthesis, ${ }^{14}$ although this is not routinely recommended. This can be done either intraoperatively or some days after the operation.

Another possibility for TV replacement surgery in the case of a pre-existing permanent pacemaker lead is to place it between the native annulus and the sewing ring of the TV, but this technique may induce a small paravalvular leak and may lead to a difficult lead removal in the future, if required. ${ }^{12,15}$

The current study has some limitations, not only because of its retrospective nature. Our patient population is small because of the rarity of patients requiring isolated reoperative TV surgery. The patient group is also very heterogeneous, which complicates comparisons of subgroups. The status "elective" or "urgent" indication for surgery was prospectively given by the surgeon at the point of surgery on the basis of the patient's clinical aspect and is partially subjective. However, these limitations apply to all retrospectively isolated TV surgery series. Follow-up time is only 2.6 years on average, and we were not able to present a complete echocardiographic follow-up after discharge. Such information would have been particularly interesting for patients with TV repair. Despite these limitations, to the best of our knowledge the current series is one of the largest of isolated reoperative TV surgery to date.

We conclude that TV surgery as a reoperation can be done with good clinical outcomes if performed electively. Minimally invasive surgery through a right lateral minithoracotomy is a safe approach for these patients.

\section{References}

1. Pinon M, Pinho P, Almeida J, Bastos P. [Isolated surgery of tricuspid insufficiency in valvular reoperation]. Rev Port Cir Cardiotorac Vasc. 2005;12:11-4. 
2. Staab ME, Nishimura RA, Dearani JA. Isolated tricuspid valve surgery for severe tricuspid regurgitation following prior left heart valve surgery: analysis of outcome in 34 patients. J Heart Valve Dis. 1999;8:567-74.

3. Bernal JM, Morales D, Revuelta C, Llorca J, Gutierrez-Morlote J, Revuelta JM. Reoperations after tricuspid valve repair. J Thorac Cardiovasc Surg. 2005;130:498-503.

4. McCarthy PM, Bhudia SK, Rajeswaran J, Hoercher KJ, Lytle BW, Cosgrove DM, et al. Tricuspid valve repair: durability and risk factors for failure. J Thorac Cardiovasc Surg. 2004;127:674-85.

5. Fehske W, Omran H, Manz M, Kohler J, Hagendorff A, Luderitz B. Color-coded Doppler imaging of the vena contracta as a basis for quantification of pure mitral regurgitation. Am J Cardiol. 1994;73:268-74.

6. Seeburger J, Borger MA, Passage J, Misfeld M, Holzhey D, Noack T, et al. Minimally invasive isolated tricuspid valve surgery. J Heart Valve Dis. 2010;19: 189-92; discussion 193.

7. Park CK, Park PW, Sung K, Lee YT, Kim WS, Jun TG. Early and midterm outcomes for tricuspid valve surgery after left-sided valve surgery. Ann Thorac Surg. 2009;88:1216-23.

8. Sung K, Park PW, Park KH, Jun TG, Lee YT, Yang JH, et al. Is tricuspid valve replacement a catastrophic operation? Eur J Cardiothorac Surg. 2009;36:825-9.
9. Nath J, Foster E, Heidenreich PA. Impact of tricuspid regurgitation on long-term survival. J Am Coll Cardiol. 2004;43:405-9.

10. Fukuda W, Aoki C, Daitoku K, Fukuda I. Vacuum-assisted venous drainage in tricuspid valve re-replacement. Interact Cardiovasc Thorac Surg. 2011;13: 101-3.

11. Kunadian B, Vijayalakshmi K, Balasubramanian S, Dunning J. Should the tricuspid valve be replaced with a mechanical or biological valve? Interact Cardiovasc Thorac Surg. 2007;6:551-7.

12. Pfannmüller B, Hirnle G, Seeburger J, Davierwala P, Schroeter T, Borger MA et al. Tricuspid valve repair in the presence of a permanent ventricular pacemake lead. Eur J Cardiothorac Surg. 2010;39:657-61.

13. Seeburger J, Merk DR, Holzhey D, Borger MA, Doll N, Mohr FW. The coronary sinus: a versatile option for pacemaker implantation during minimally invasive valve surgery. J Card Surg. 2009;24:431-2.

14. Antonelli D, Freedberg NA. Endocardial ventricular pacing through a bioprosthetic tricuspid valve. Pacing Clin Electrophysiol. 2007;30:271-2.

15. Aris A, Callejo F, Cobiella J, Maestre ML. Tricuspid valve replacement in the presence of an endocardial pacemaker electrode. J Heart Valve Dis. 2004;13: $523-4$. 